Obviously, $\nabla_{n}=V_{n}^{-1}$ and $\lambda\left(V_{n}\right)>0 \quad(n=1,2, \ldots)$. Further, we define the sequence of symmetric probability distributions $\mu_{1}, \mu_{2}, \ldots$ by the following formula:

$$
\mu_{n}(E)=\frac{1-a_{n}+a_{n} \lambda\left(V_{n}\right)}{\lambda\left(V_{n}\right)} \lambda\left(E \cap V_{n}\right)+a_{n} \lambda\left(E \backslash V_{n}\right) \quad(n=1,2, \ldots) .
$$

It is easy to verify that the inequality

$$
\mu_{n}(E) \geqslant a_{n} \lambda(E) \quad(n=1,2, \ldots)
$$

is true for every Borel subset $E \subset G$. Moreover, in virtue of (1.6),

$$
\begin{aligned}
&\left\|\varphi_{\mu_{n}}\left(\mathcal{U}_{0}\right)-\varepsilon\right\| \leqslant \int_{G}\left\|\mathcal{U}_{0}(g)-\mathcal{E}\right\| \mu_{n}(d g) \\
&= \frac{1-a_{n}+a_{n} \lambda\left(V_{n}\right)}{\lambda\left(V_{n}\right)} \int_{V_{n}}\left\|\mathcal{U}_{0}(g)-\varepsilon\right\| \lambda(d g)+a_{n} \int_{G \backslash V_{n}}\left\|\mathcal{U}_{0}(g)-\varepsilon\right\| \lambda(d g) \leqslant n^{-2}+2 a_{n} \\
&(n=1,2, \ldots) .
\end{aligned}
$$

Hence, according to (15), we get the inequality

$$
\sum_{n=1}^{\infty}\left\|\varphi_{\mu_{n}}\left(\mathcal{U}_{0}\right)-\varepsilon\right\|<\infty
$$

which implies the convergence

$$
\lim _{n \rightarrow \infty} \varphi_{\mu_{1}{ }^{*} \mu_{2} * \ldots \mu_{n}}\left(\mathcal{U}_{0}\right)=\lim _{n \rightarrow \infty} \varphi_{\mu_{1}}\left(\mathcal{U}_{0}\right) \varphi_{\mu_{2}}\left(\mathcal{U}_{0}\right) \ldots \varphi_{\mu_{n}}\left(\mathcal{U}_{0}\right) \neq 0 .
$$

Consequently, the Haar measure is not the weak limit of the probability distributions $\mu_{1} * \mu_{2} * \ldots * \mu_{n}(n=1,2, \ldots)$. The Theorem is thus proved.

INSTITUTE OF RADIO-ENGINEERING AND ELECTRONICS,

CZECHOSLOVAK ACADEMY OF SCIENCES

MATHEMATIOAL INSTITUTE OF THE POLISH ACADEMY OF SCIHNCES

Reģu par la Rédaction le 23. 3. 1959

\begin{tabular}{lll}
\hline VOL. VII & 1960 & FASC. 2 \\
\hline
\end{tabular}

\section{ON THE POWER OF COMPACT SPACES}

$\mathrm{BY}$

A. HULANICKI (WROCEAW)

Let $X$ be a compact (= bicompact) infinite space. The character $\Theta(p)$ of a point $p \in X$ is the least cardinal of a family of open sets containing the point $p$ and having that point as its intersection.

Recently S. Mrówka [4] has shown the following

THEOREM. If $\mathfrak{m} \leqslant \Theta(p)$ for all $p \in X$, then $\overline{\bar{X}} \geqslant 2^{\mathfrak{n t}}$.

In this note we present a somewhat simpler proof of this theorem. The main idea of the proof is similar to that of my papers [1] and [2] and has some connections with the idea used by F. B. Jones in [3].

Proof. Denote by $I^{a}$ the set of all $0-1$ sequences of the ordinal type $a$. Let $\xi \in I^{\alpha}$ and $\beta<\alpha$. Denote by $\xi_{\beta}$ the segment of the type $\beta$ of the sequence $\xi$. For each $\alpha$ we are going to define a family $\left\{V(\xi): \xi \in I^{a}\right\}$ of open sets of the space $X$. We use the transfinite induction: for $a=1$ we have two one-element sequences 0 and 1 . Let $V(0)$ and $V(1)$ be two arbitrary disjoint open subsets of $X$. Suppose we have defined $\nabla(\xi)$ for all $\xi \in I^{\beta}$ and $\beta<a$. In order to define $V(\xi)$ for $\xi \in I^{\alpha}$ consider two possibilities: (a) $\alpha$ has a precedent and (b) $\alpha$ is a limit-number.

(a) Put $\alpha=\alpha^{\prime}+1$. Then either for some $\xi \in I^{\alpha^{\prime}}$ the set $\bigcap_{\beta \leqslant a^{\prime}} V\left(\xi_{\beta}\right)$ contains at most one point or for every $\xi \in I^{a^{\prime}}$ it consists of two points at least. In the first case, the sets $V(\xi)$ for $\xi \in I^{\alpha}$ will not be defined. In the second case, given a $\xi \in I^{\alpha^{\prime}}$, let $x_{0}$ and $x_{1}$ be two points of $\bigcap_{\beta \leqslant a^{\prime}} V\left(\xi_{\beta}\right)$.

There are exactly two different sequences $\eta^{0}$ and $\eta^{1}$ belonging to $I^{a}$ such that $\eta_{a^{\prime}}^{0}=\eta_{a^{\prime}}^{1}=\xi$. Let $V\left(\eta^{0}\right)$ and $V\left(\eta^{1}\right)$ be two disjoint open sets such that $x_{0} \epsilon V\left(\eta^{0}\right), x_{1} \in V\left(\eta^{1}\right)$ and $\overline{V\left(\eta^{0}\right)} \subset V(\xi), \overline{\nabla\left(\eta^{1}\right)} \subset V(\xi)$.

(b) If $\alpha$ is a limit-number, then for $\xi \in I^{\alpha}$ put $V(\xi)=X$.

Note that:

(i) For every $\alpha$ the set $\bigcap_{\beta \leqslant a} V\left(\xi_{p}\right)$ is non-void. 
COLLOQUIUM MATHEMATICUM

It is obvious if $\alpha$ has a precedent, for $\alpha$ being a limit-number, it follows from the equality

$$
\bigcap_{\beta \leqslant a} \overline{V\left(\xi_{p}\right)}=\bigcap_{\beta \leqslant \alpha} V\left(\xi_{p}\right)
$$

and from the compactness of $X$.

(ii) If for some $\gamma$ the sets $V(\xi)$ with $\xi \in I^{\gamma}$ are defined, then the family $\left\{\bigcap_{\beta \leqslant \gamma} \nabla\left(\xi_{\beta}\right): \xi \in I^{\gamma}\right\}$ contains a subfamily of cardinal $2^{\bar{\gamma}}$ consisting of disjoint $\beta \leqslant \gamma$
sets. Indeed, if two sequences $\xi^{1}, \xi^{2} \in I^{\gamma}$ differ in the $\beta$-th position, and $\beta$ is not a limit-number, then the sets $V\left(\xi_{\beta}^{1}\right)$ and $V\left(\xi_{\beta}^{2}\right)$ are disjoint; hence $\bigcap_{\beta \leqslant \gamma} V\left(\xi_{\beta}^{1}\right)$ and $\bigcap_{\beta \leqslant \gamma} V\left(\xi_{\beta}^{2}\right)$ are disjoint.

Let $\gamma$ be the first ordinal number such that the set $V(\xi)$ is not defined for $\xi \in I^{\gamma+1}$. Then for some $\xi \in I^{\gamma}$ the set $\bigcap_{\beta \leqslant \gamma} V\left(\xi_{\beta}\right)$ contains less than two elements. Hence, by (i), we get

$$
\bigcap_{\beta \leqslant \gamma} V\left(\xi_{p}\right)=(p) .
$$

The last equality implies $\Theta(p) \leqslant \bar{\gamma}$ and, if $\Theta(p) \geqslant \mathfrak{m}$, we get, by (ii), $\overline{\bar{X}} \geqslant 2^{\bar{\gamma}} \geqslant 2^{\Theta(p)} \geqslant 2^{\mathfrak{m}}$.

\section{REFERENOES}

[1] A. Hulanicki, On locally compact topological groups of power of continuum, Fundamenta Mathematicae 44 (1957), p. 156- 158.

[2] - On cardinal numbers related with locally compact groups, Bulletin do l'Académie Polonaise des Sciences, Série des Sciences Mathématiques, Astronomi ques et Physiques, 6 (1958), p. 67-70.

[3] F. B. Jones, On the first countability axiom for locally compact Hausdorff spaces, Colloquium Mathematicum 7 (1959), p. 33-34.

[4] S. Mrówka, A generalization of a theorem concerning the power of a perfect compact metric space, Bulletin de I'Académie Polonaise des Sciences, Sério des Sciences Mathématiques, Astronomiques et Physiques, 6 (1958), p. 89.93.

Mathematical institute of the POLISH ACADEMY OF scifencies

Regu par la Rédaction le 29. 12. 1958

VOL. VII

1960

FASC. 2

SUPPLÉ MENT À L'ARTICLE

„SUR DEUX PROBLËMES D'ANALYSE NON RÉSOLUS"

PAR

M. FRÉCHET (PARIS)

I. Une modification d'une démonstration. Dans la réciproque de la p. 39 de mon travail [1], on suppose ,que la somme de deux courbes ne soit définie que dans le cas où ces deux courbes sont des lignes polygonales orientées". Cette supposition n'ayant pas été respectée dans la démonstration, il y a lieu de modifier celle-ci de la manière suivante.

On aura

$$
\begin{aligned}
\left(Q_{\varepsilon}, Q_{\omega}\right)= & \left(P_{\varepsilon}+P_{\varepsilon}^{\prime}, P_{\omega}+P_{\omega}^{\prime}\right)=\left\|\left(P_{\varepsilon}+P_{\varepsilon}^{\prime}\right)+(-1)\left(P_{\omega}+P_{\omega}^{\prime}\right)\right\| \\
= & \left\|\left(P_{\varepsilon}+(-1) P_{\omega}\right)+\left(P_{\varepsilon}^{\prime}+(-1) P_{\omega}^{\prime}\right)\right\| \\
\leqslant & \left\|P_{\varepsilon}+(-1) P_{\omega}\right\|+\left\|P_{\varepsilon}^{\prime}+(-1) P_{\omega}^{\prime}\right\|=\left(P_{\varepsilon}, P_{\omega}\right)+\left(P_{\varepsilon}^{\prime}, P_{\omega}^{\prime}\right) . \\
& \left(P_{\varepsilon} ; P_{\omega}\right) \leqslant\left(P_{\varepsilon}, c\right)+\left(c, P_{\omega}\right) \leqslant \varepsilon+\omega .
\end{aligned}
$$

$\mathrm{Or}$

De même pour les $P^{\prime}$. On a donc

$$
\left(Q_{\varepsilon}, Q_{\omega}\right) \leqslant 2 \varepsilon+2 \omega
$$

comme dans le texte déjà paru. D'après ce même texte: „L'espace $T$, considéré comme espace distancié, (...) est complet". Dès lors, il résulte de la formule (8) que $Q_{\varepsilon}$ tend vers une courbe déterminée $\sigma$ quand $\varepsilon \rightarrow 0$. Cette courbe limite ne dépend pas du choix des lignes polygonales $P_{\varepsilon}, P_{\varepsilon}^{\prime}$. Si on leur substitue deux lignes polygonales orientées $p_{\varepsilon}, p_{\varepsilon}^{\prime}$ et si l'on pose $q_{\varepsilon}=p_{\varepsilon}+p_{\varepsilon}^{\prime}$, on aura (mais ici nous modifions encore une fois les formules):

$$
\begin{aligned}
\left(Q_{\varepsilon}, q_{\varepsilon}\right) & \equiv\left(P_{\varepsilon}+P_{\varepsilon}^{\prime}, p_{\varepsilon}+p_{\varepsilon}^{\prime}\right)=\left\|P_{\varepsilon}+P_{\varepsilon}^{\prime}+(-1)\left(p_{\varepsilon}+p_{\varepsilon}^{\prime}\right)\right\| \\
& =\left\|\left(P_{\varepsilon}+(-1) p_{\varepsilon}\right)+\left(P_{\varepsilon}^{\prime}+(-1) p_{\varepsilon}^{\prime}\right)\right\| \\
& \leqslant\left\|P_{\varepsilon}+(-1) p_{\varepsilon}\right\|+\left\|P_{\varepsilon}^{\prime}+(-1) p_{\varepsilon}^{\prime}\right\|=\left(P_{\varepsilon}, p_{\varepsilon}\right)+\left(P_{\varepsilon}^{\prime}, p_{\varepsilon}^{\prime}\right) .
\end{aligned}
$$

